\title{
Relationship among Mental Toughness, Stress Coping Styles and Optimism in Veteran and Disabled Volleyball Players
}

\section{ART ICLE INF O}

\section{Article Type}

Descriptive Study

\section{Authors}

Salman Z**PhD,

Pooraghaei Ardekani Z. ${ }^{1} P h D$,

Hossein Fahimi T. ${ }^{1} M S C$

How to cite this article
Salman Z, Pooraghaei Ardekani Z,
Hossein Fahimi T. Relationship
among Mental Toughness, Stress
Coping Styles and Optimism in
Veteran and Disabled Volleyball
Players. Iranian Journal of War
\& Public Health. 2017;9(1):9-14.

*Motion Behavior Department, Physical Education \& Sport Sciences Faculty, Allameh Tabataba'i University, Tehran, Iran

${ }^{1}$ Motion Behavior Department, Physical Education \& Sport Sciences Faculty, Allameh Tabataba'i University, Tehran, Iran

\section{Correspondence}

Address: Physical Education \& Sport Sciences Faculty, In Front of the Olympic Hotel, Hakim Highway West, Tehran. Postal Code: 1485743411

Phone: +98 (21) 44118630

Fax: +98 (21) 44118629

z_salman2005@yahoo.com

\section{Article History}

Received: May 17, 2016

Accepted: November 19, 2016

ePublished: March 10, 2017

\section{A B S T R A C T}

Aims Many and different tensions that the veteran and disabled athletes always experience during their lives take part in their mental activities. Therefore, one of the most effective methods in the prevention of their mental disorders is to find out the psychological factors that resolve the veteran athletes' behavioral disorders and problems. The aim of the study was to determine the correlation between mental toughness, the stress coping styles, and optimism in the veteran and disabled athletes of the Iranian Sitting Volleyball Super-League.

Instrument \& Methods In the correlational descriptive study, 70 male veteran and disable volleyball athletes of the Iranian Sitting Volleyball Super-League were studied in 2014. The subjects were purposefully selected. Data was collected by athlete mental toughness, stress coping styles, and the life-orientation revised test questionnaires. Data was analyzed by SPSS 20 software using correlational Spearman correlation and simple linear regression tests.

Findings The mental toughness significantly correlated with the stress coping styles and the problem-focused, intuitional, and avoidance styles. More than $8 \%$ of the changes in the stress coping styles was explained by the mental toughness. The optimism significantly correlated by the mental toughness and the confidence and control components. In addition, more than $18 \%$ of the mental roughness changes was explained by the optimism. However, the optimism significantly correlated with the intuitional stress coping style only.

Conclusion The tougher veteran and disabled volleyball athletes cope with stress through better styles. In addition, it is possible to improve mental toughness through the optimism skills trainings.

Keywords Mental Deficiency; Optimism; Coping Skills; Volleyball

\section{I T A T I O N L I N KS}

[1] The relationships between mental toughness and physical fitness in ... [2] Progress toward construct validation of the sports mental toughness questionnaire ... [3] What is this thing called mental toughness? ... [4] What is this thing called mental toughness? ... [5] Mental toughness: The concept and its... [6] Mental toughness, optimism, and coping among ... [7] Psychometric properties of the sport stress coping styles ... [8] Exploring gender equivalence and bias in a measure of psychological ... [9] The relationship between coping strategies and resilience and optimism in the city of Dezful ... [10] Psychological characteristics and their development in Olympic ... [11] Dispositional optimism and coping: A meta-analytical ... [12] The relationship of resiliency and selfconcept with self-efficacy of handicapped females: the mediating ... [13] Emotional intelligence in athletes ... [14] Are elite athletes with disabilities mentally tougher ... [15] Comparing men and women athletes mentally tough individual contact and non-contact disciplines ... [16] Factorial validity of the coping scale for Korean ... [17] The styles of coping with stress in team and individual athletes based on gender ... [18] Optimism coping, and health: Assessment and implications ... [19] On the power of positive thinking: The benefits ... [20] Optimism mediating role in the relationship between educational experience and respond ... [21] Optimism-pessimism and coping strategies: prediction of ... [22] The relationship between creativity and emotional intelligence to cope with stress ... [23] The domain of psychological researches [24] Mind over matter: The development of the mental ... [25] Moderating variables in the relationship between mental ... [26] Cognitive appraisals in sport: The direct and moderating role ... [27] Sources of stress experienced by elite male wheelchair ... [28] The relationship between optimism and the secretion of ... [29] The relationship between coping style and mental ... [30] An inquiry into the relationship of islamic optimism ... 
مقابله با فشارها و سختىها، عبور از موانع و شكست ها، تمركز بر

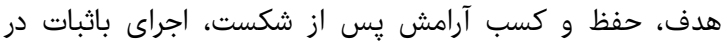

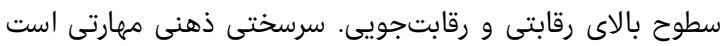

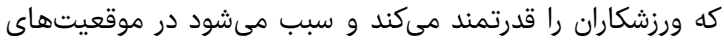

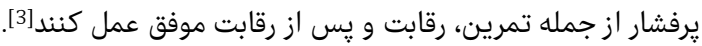

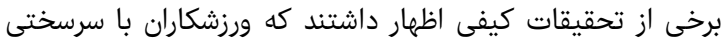

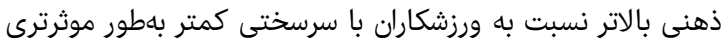

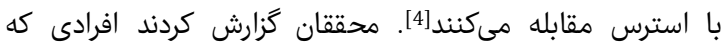

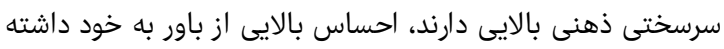

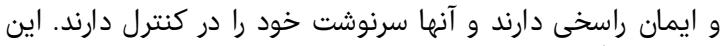

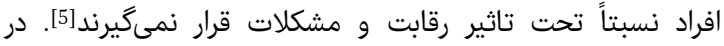

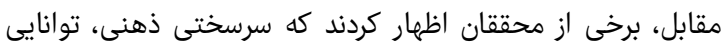

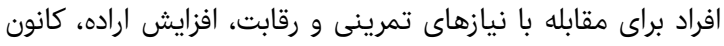

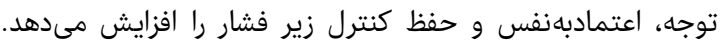

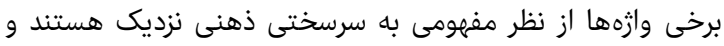

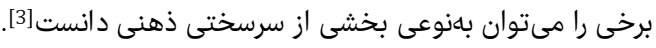

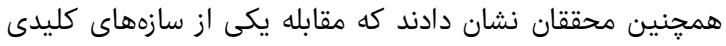

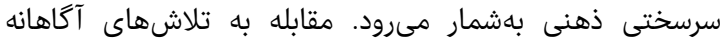

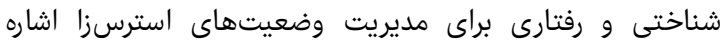

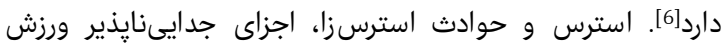

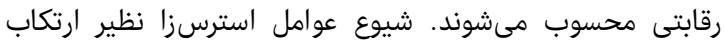

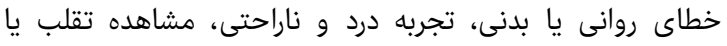

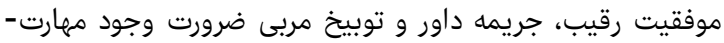

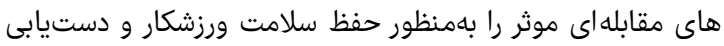

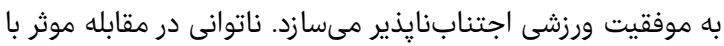

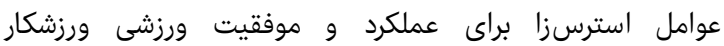

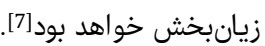

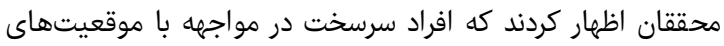

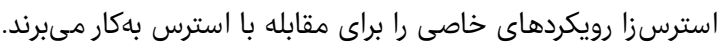

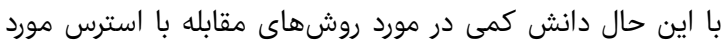

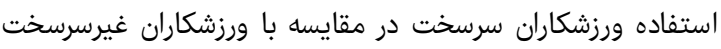

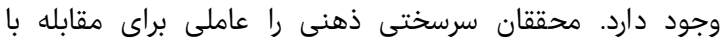

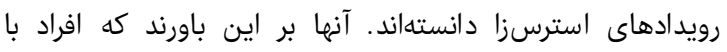

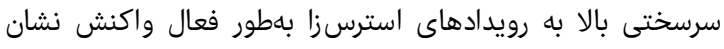

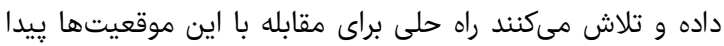

كنند [8]

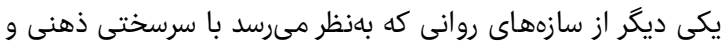

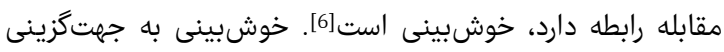

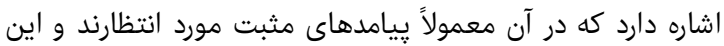

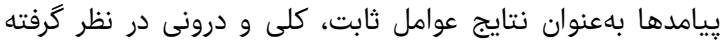

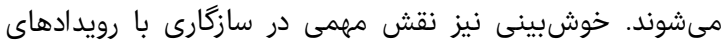

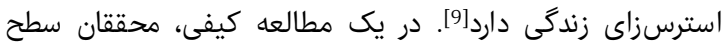

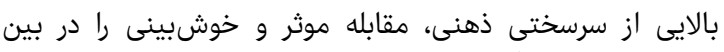

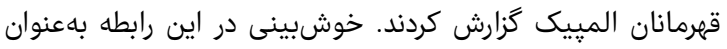

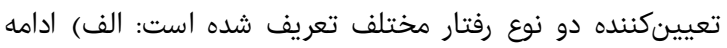

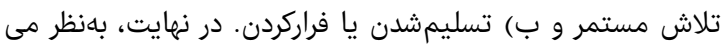

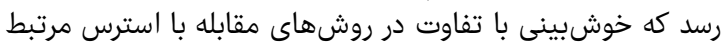

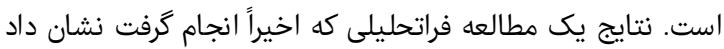

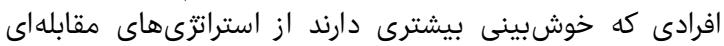

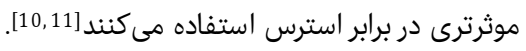

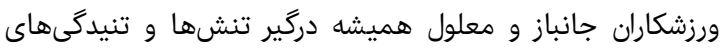

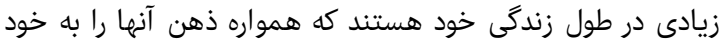

دوره 9، شماره ا، زمستان هوس1
رابطه ميان سرسختى ذهنى، روشهاي مقابلى مقابله با استرس و خوشبينى در بازيكنان واليبال جانباز و معلول مابل

PhD زهرا سلمان

كروه رفتار حركتى، دانشكده تربيت بدانى و و علوم ورزشى، دانشكاه علامه

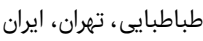

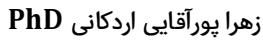

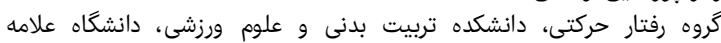
طباطبايى، تهران، ايران

طه حسين فهيمى MSc كروه رفتار حركتى، دانشكده تربيت بدرئ بدنى و علوم ورزشى، دانشكاه علامه طباطبايى، تهران، ايران

جكيده اهداف: ورزشكاران جانباز و معلول هميشه دركير تنشهاى زئن زيادى در

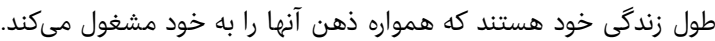

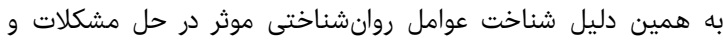

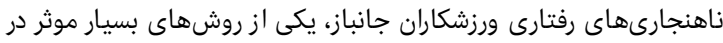

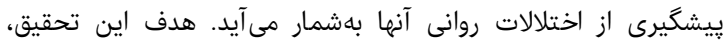

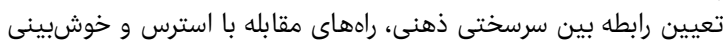

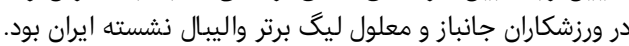

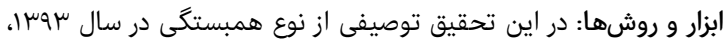

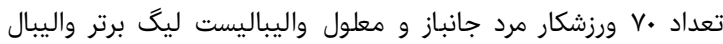

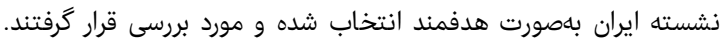

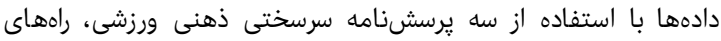

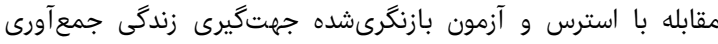

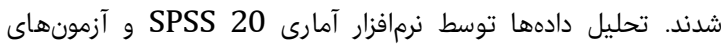

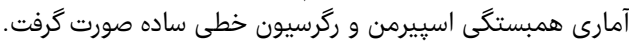

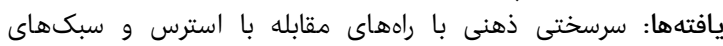

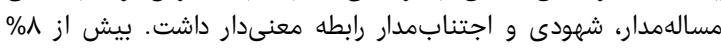

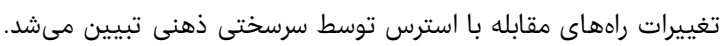

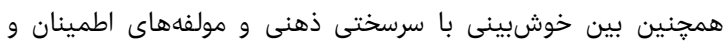

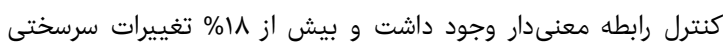

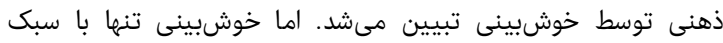
شهودى مقابله با استرس رابطه معنى تودار داشتئ.

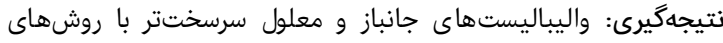

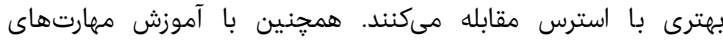

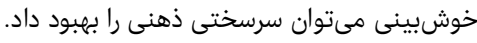

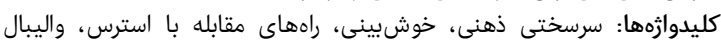

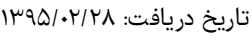

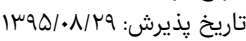

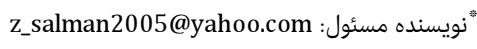

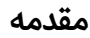

امروزه ويزگىهاى رواندهان رواناختى در موقعيتهاى تحت فشار و

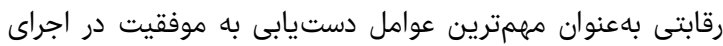

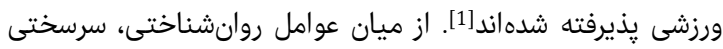

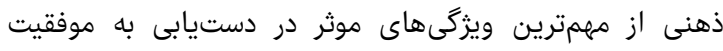

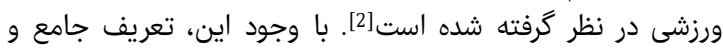

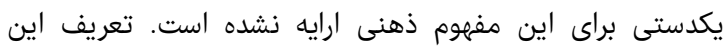

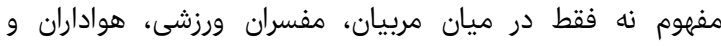

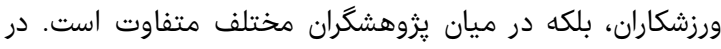

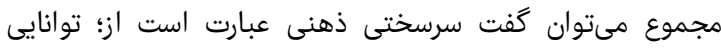




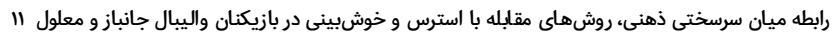

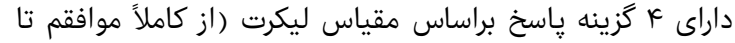

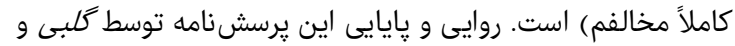

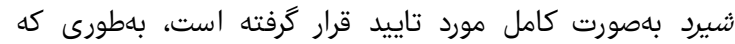

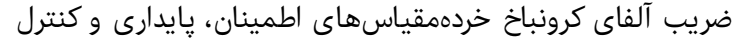

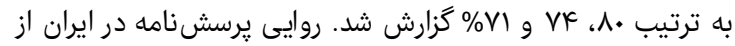

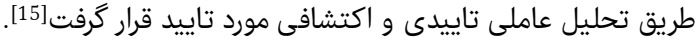

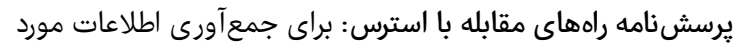

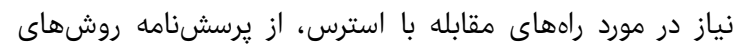

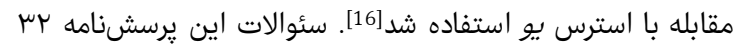

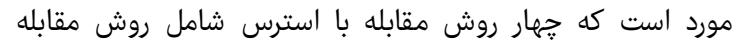

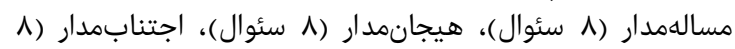

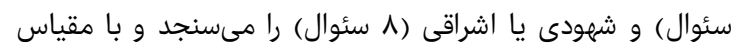

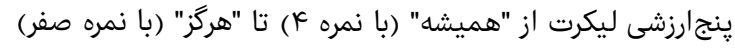

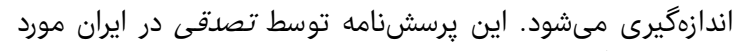

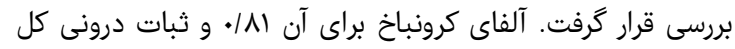

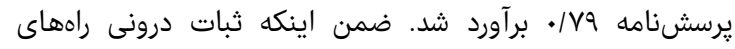

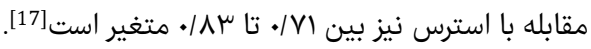

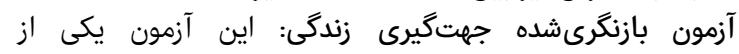

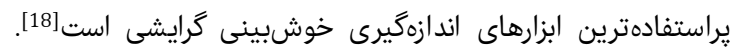

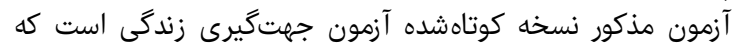

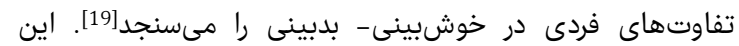

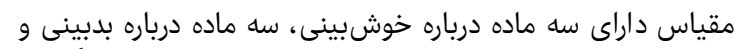

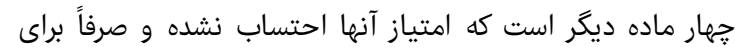

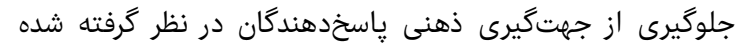

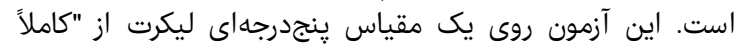

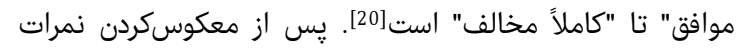

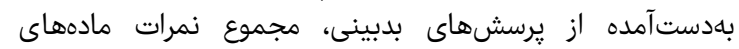

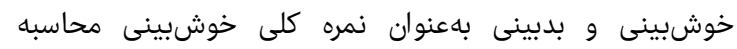

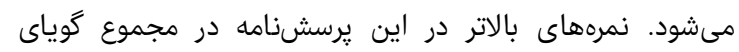

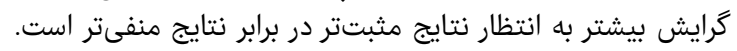

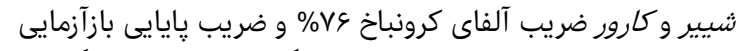
\%Vq

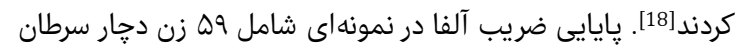

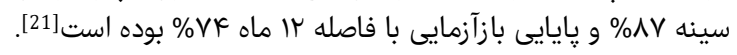

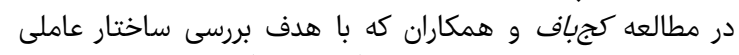

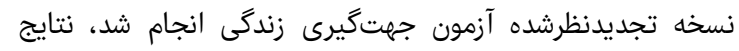

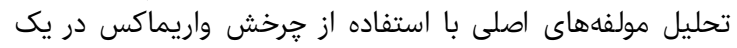

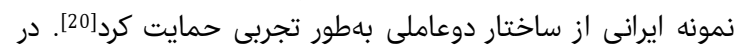

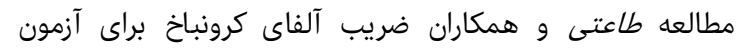

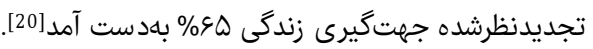

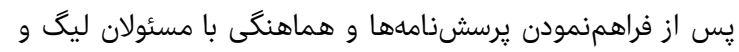

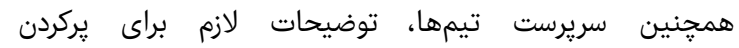

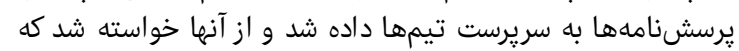

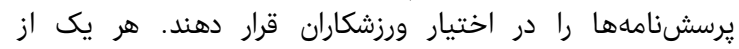

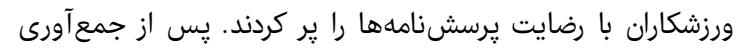

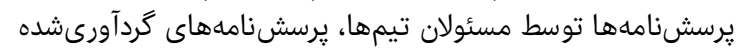

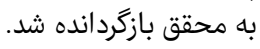

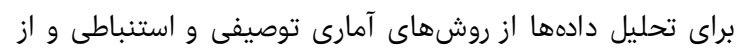

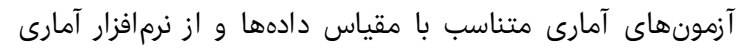

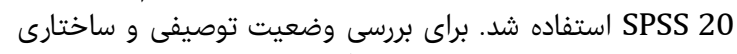

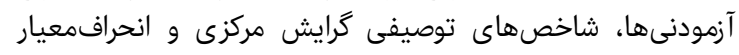

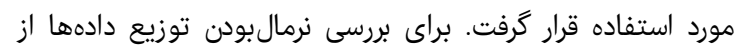

Iranian Journal of War and Public Health

\section{年}

مشغول مىكند و در اكثر موارد ناتوانى آنها زير سئوال مىرود،

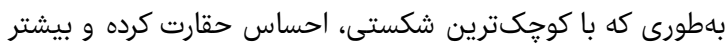

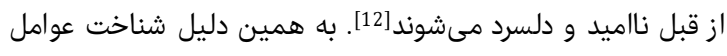

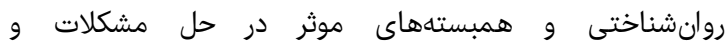

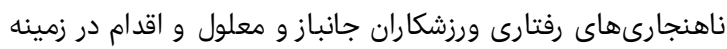

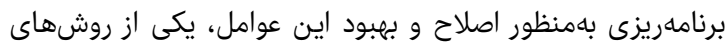

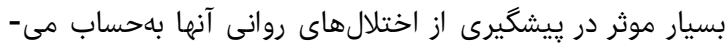

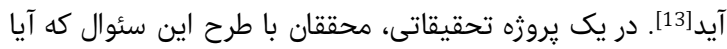

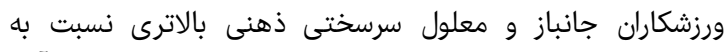

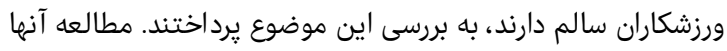

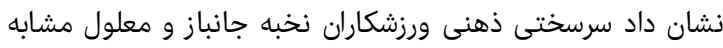

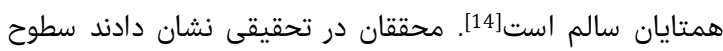

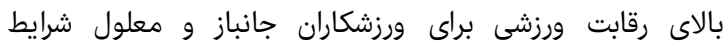

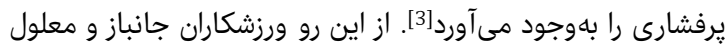

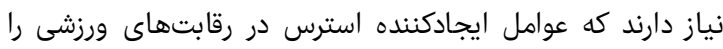

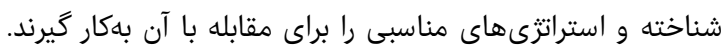

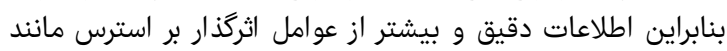

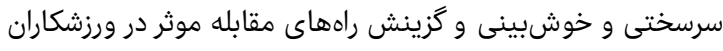

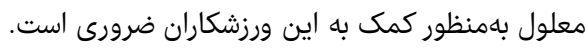

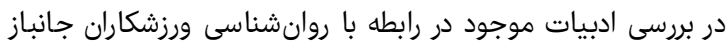

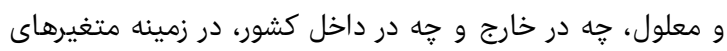

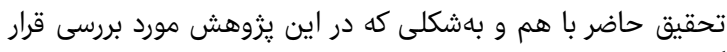

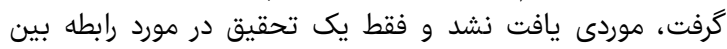

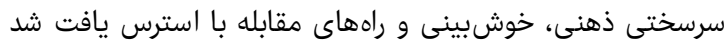

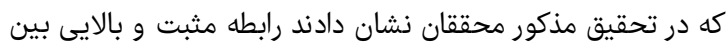

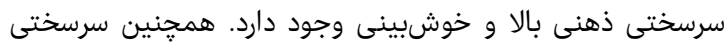

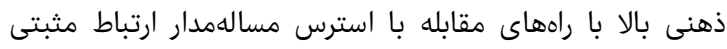

دارد[6].

بهطور خلاصه، سرسختى ذهنى يك سازه روان شناسى جذاب براى

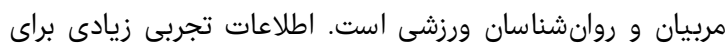

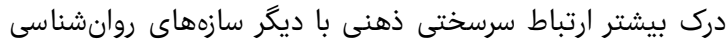

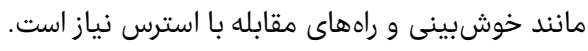

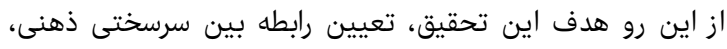

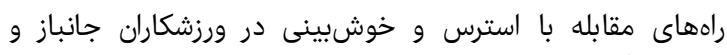

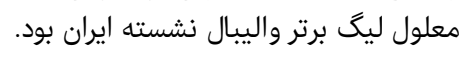

\section{ابزار و روشها}

اين تحقيق توصيفى از نوع همبستخى است كه در سال سوسا در

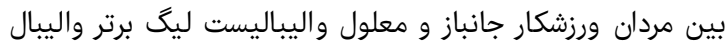

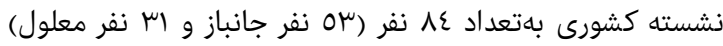

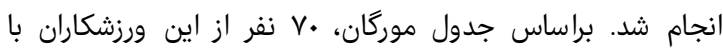

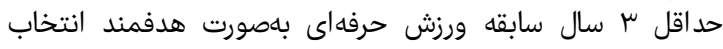

شدند.

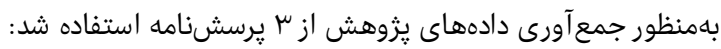

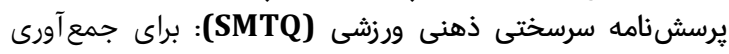

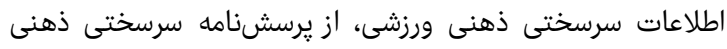

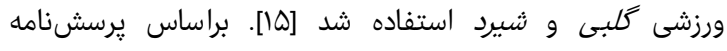

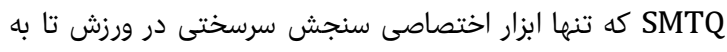

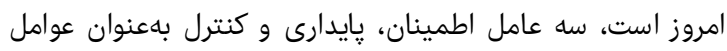

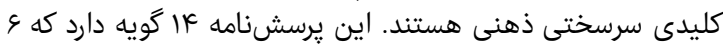

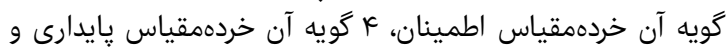

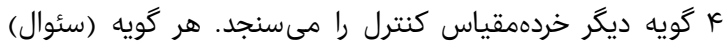

Volume 9, Issue 1, Winter 2017 
سرسختى ذهنى با سبك هيجانمدار مقابله با استرس رابطه

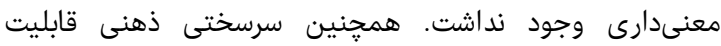
ييشبينى 1\%از راههاى مقابله با استرس را داشت

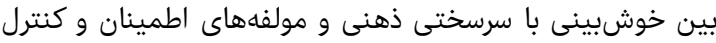

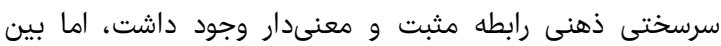

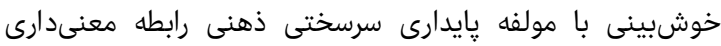

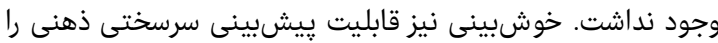

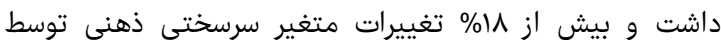

متغير خوشبينى تبيين مى خئد

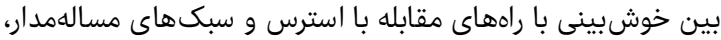

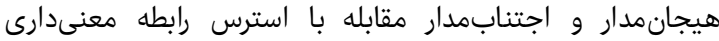

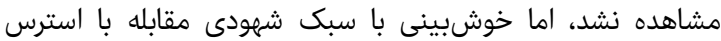

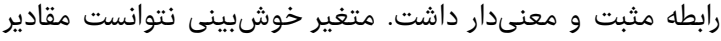

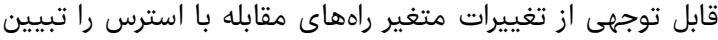

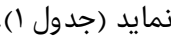

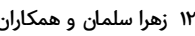

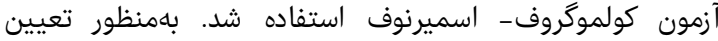

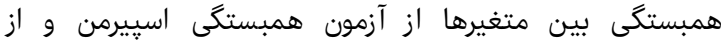

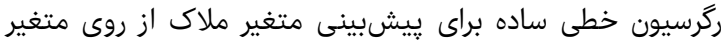

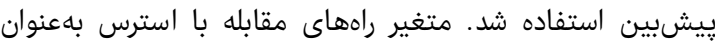

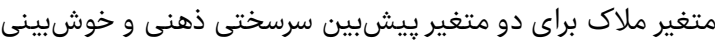

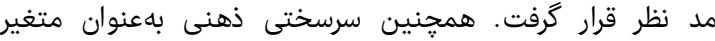

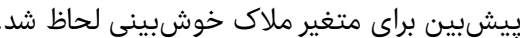

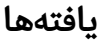

ميانكين سنى شركتكنندگان در يزوهش

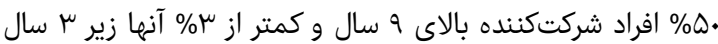
سابقه ورزش حرفهاى داشتند.

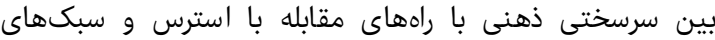

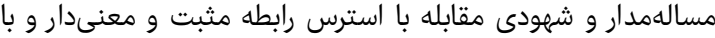

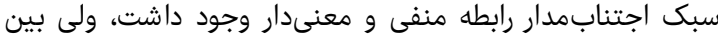

\begin{tabular}{|c|c|c|c|c|c|c|c|c|c|}
\hline 9 & $\Lambda$ & $\mathrm{V}$ & 7 & 0 & $\varepsilon$ & $\mu$ & $r$ & 1 & متغيرها \\
\hline & & & & & & & & 1 & ا - سرسختى ذهنى \\
\hline & & & & & & & 1 & $\cdot / \wedge \Lambda *^{* *}$ & r - مولفه اطمينان \\
\hline & & & & & & 1 &. $\mid \varepsilon \mu \gamma^{* *}$ & $\cdot / \Lambda \cdot \Lambda^{* *}$ & 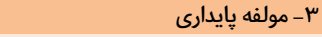 \\
\hline & & & & & 1 & $\cdot / \mu_{\wedge} \Lambda^{* *}$ & $\cdot / k \mu . * *$ & $\cdot / \vee \cdot \Lambda^{* *}$ & ع - مولفه كنترل \\
\hline & & & & 1 &.$/ \ll \& \Lambda^{* *}$ & . ITE &.$/ K V \varepsilon^{* *}$ &.$/ 4 \wedge q^{* *}$ & 0- خوشبينى \\
\hline & & & 1 &.$/ 1{ }^{\mu} k$ & $\cdot 1 \cdot 11$ & $\cdot \mid Y K I^{*}$ & $\cdot|Y M|$ & $\cdot /\left.Y V\right|^{*}$ & 7 - راههاى مقابله با استرس \\
\hline & & 1 &.$/ K \vee q^{* *}$ & $\cdot / K I \Lambda$ & $\cdot / \mu r \cdot * *$ & $\cdot \mid \Delta S V^{* *}$ &.$/ 499^{* *}$ & $\cdot \mid \Delta \& \mu^{* *}$ & V - سبك مسالهمدار \\
\hline & 1 & $\cdot / M \mid \Lambda$ & $\cdot / \Delta \Lambda^{* *}$ &.$- / 194$ &.$- / 1 k 4$ &.$/ .19$ &.$/ .19$ &..$- \cdot 1 \mu$ & A- سبك هيجانمدار \\
\hline 1 &.$\cdot \mu r$ & $-\cdot / 4 \cdot 9^{* *}$ & $\cdot / N^{\prime} \mid V^{* *}$ &.$- / 11 \mu$ & $-\cdot / r V I^{*}$ &.$- / \mu 1 q^{* *}$ &.$- / Y F \Lambda^{*}$ & $-\bullet / r^{*} r^{*}$ & 9- سبك اجتنابمدار \\
\hline.$/ 119$ & .1 .94 & $\cdot / 1 \cdot \mu$ & $.19 \Delta Q^{* *}$ & *.ITKS &.$/ 199$ &.$/ 198$ & $\cdot / r . \cdot$ & $\cdot / 44 . *$ & •ا - سبك شهودى \\
\hline
\end{tabular}

مىتواند با عوامل استرسزا مقابله كند[23] و بهدليل اينكه افراد

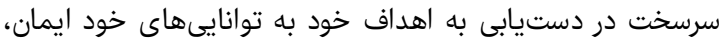

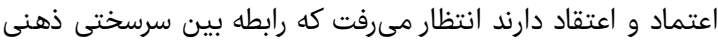

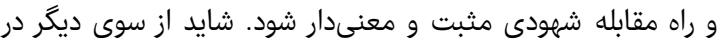

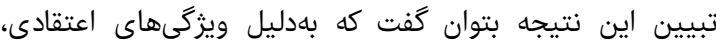
اسلامى و مذهبى نمونه تحقيق رابطه بين سرسختى ذئن ذهنى و راه

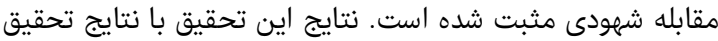

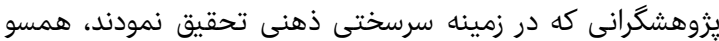

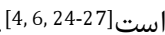

رابطه بين سرسختى ذهنى با مولفه اجتنابمدار راههاى مقابله با

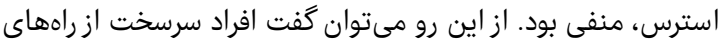

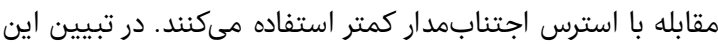

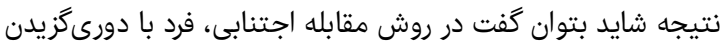

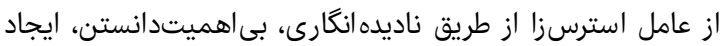

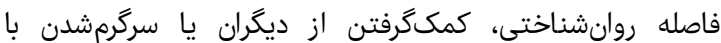

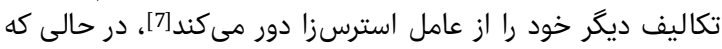

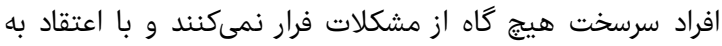

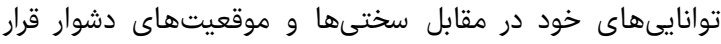
مى ئيرند و سعى در كنترل موقعيت ها دارئ دارند.

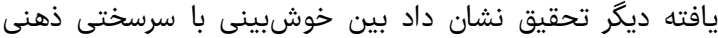

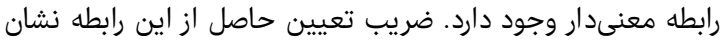

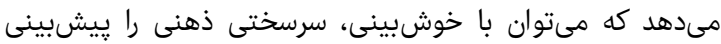

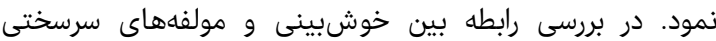
ذهنى، نتايج بهدستآمده نشان داد بين خوشبينى

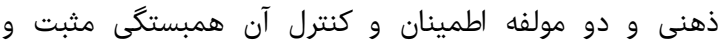

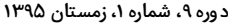

بحث

تحقيق حاضر با هدف بررسى رابطه بين سرسختى ذهنى، راههاى

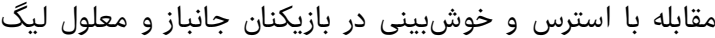

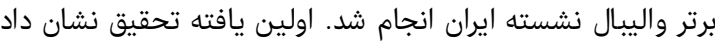
بين سرسختى ذهنى و راههاى مقابله با استرس رابطه معنى دإدار

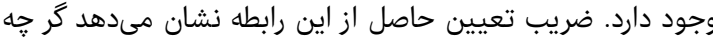

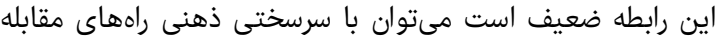

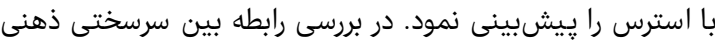

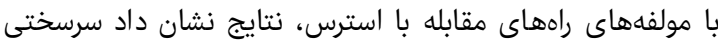

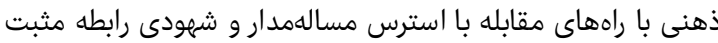

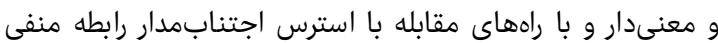

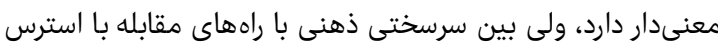

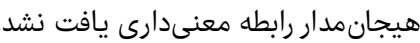

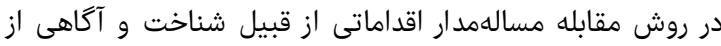

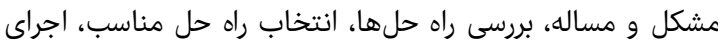

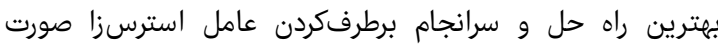

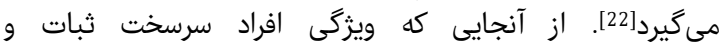

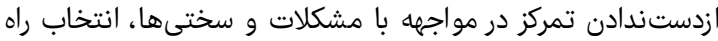

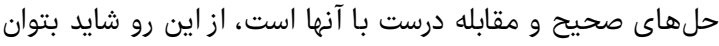

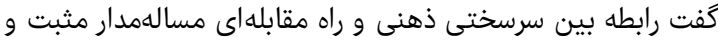
معنى دار شده است.

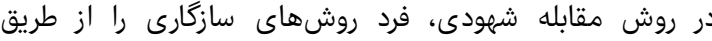

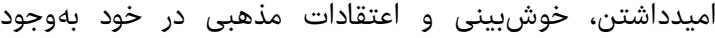

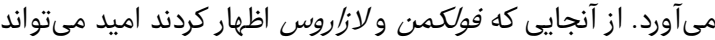

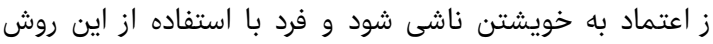


رابطه ميان سرسختى ذهنى، روشهاى مقابله با استرس و خوشبينى در بازيكنان واليبال جانباز و معلول سرا

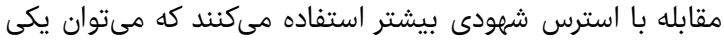

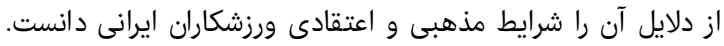

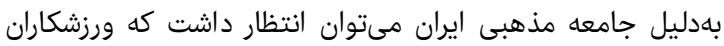

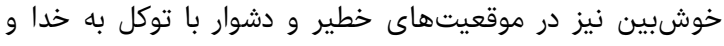

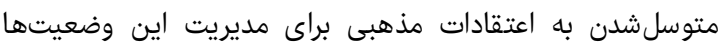
سعى كنند.

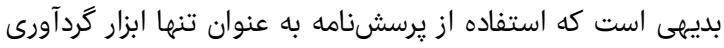

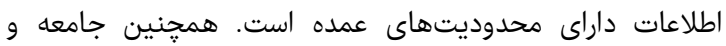

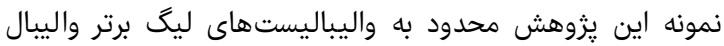

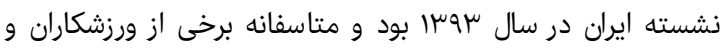

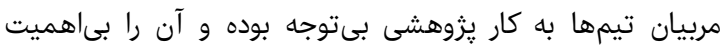

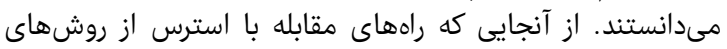

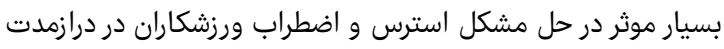

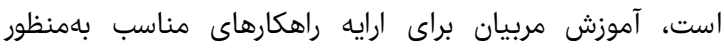

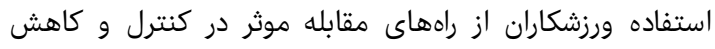

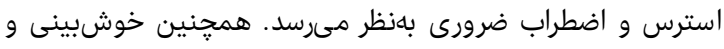

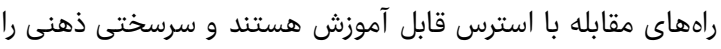

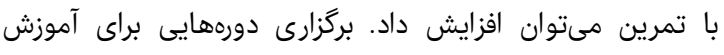

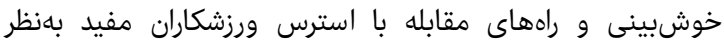

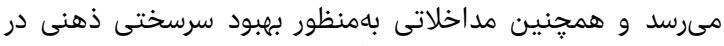
تمرينات تيمها را مىتوان در نظر گرفت

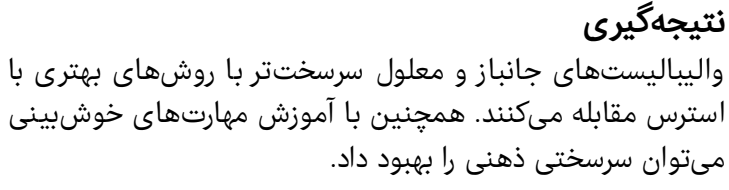

تشكر و قدردانى: از تمامى دوستان و عزيزانى كه ما را در اجراى اين

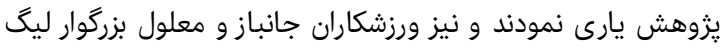

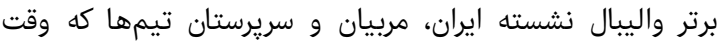

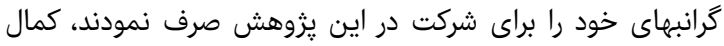

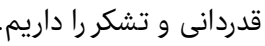
تاييديه اخلاقى: براى شركت آزمودنى ها در تحقيق آنديق، فرم رضايتنامه

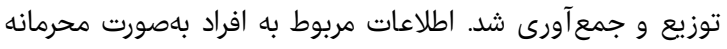

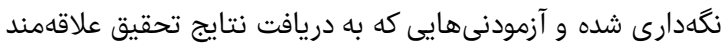

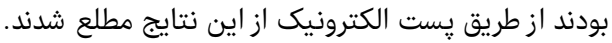

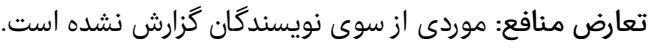

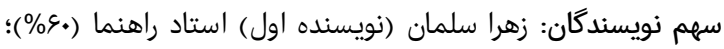

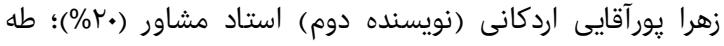

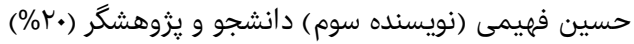

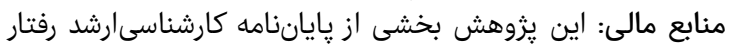

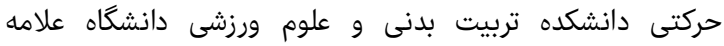
طباطبايى است.

منابع 1- Hatami F, Hatami Shahmir E, Adibpur N, Tahmasebi F. The relationships between mental toughness and physical fitness in female students. J Sport Psychol Stud. 2012;2(4):53-64. [Persian]

2- Sheard M, Golby J, van Wersch A. Progress toward construct validation of the sports mental toughness questionnaire (SMTQ). Eur J Psychol Assess. 2009;25(3):186-93.

3- Jones G. What is this thing called mental toughness? an
متوسطى وجود دارد. در تبيين اين يافتهها شايد بتوان گفت از نظر

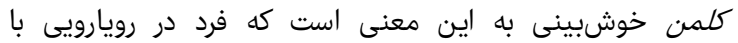

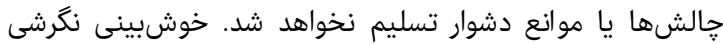

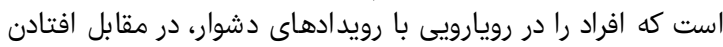

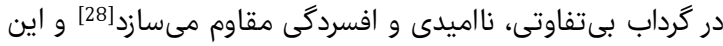

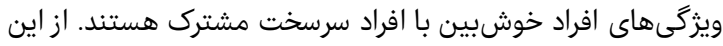

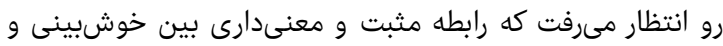

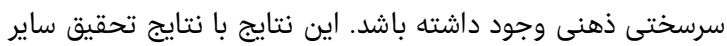

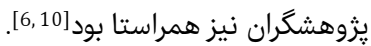

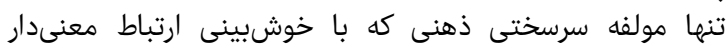

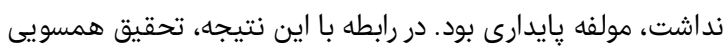

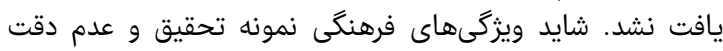

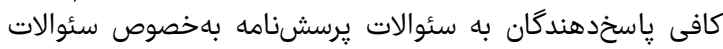

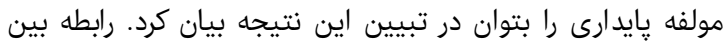

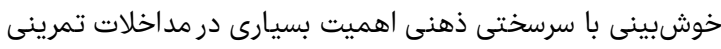

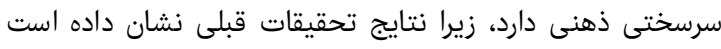

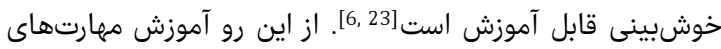

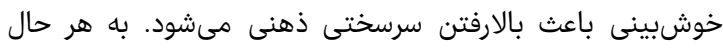

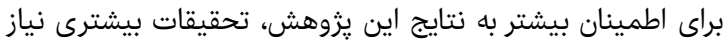

است.

يافته ديكر تحقيق نشان داد بين خوشبينى و راههاى مقابله با

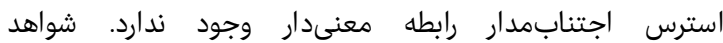

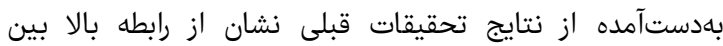

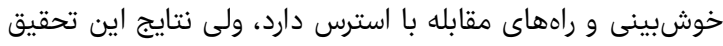

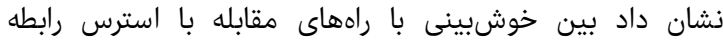

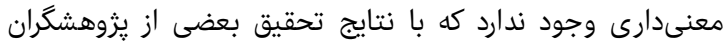

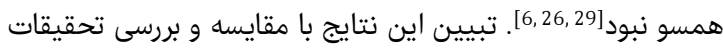

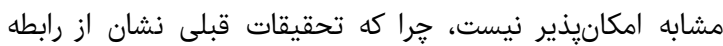

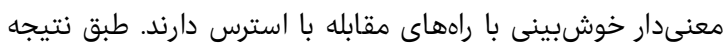

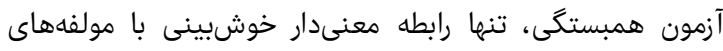

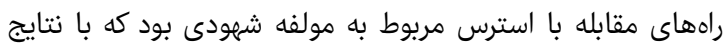

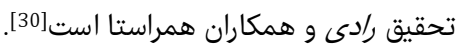

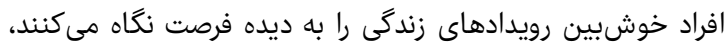

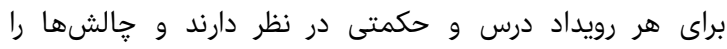

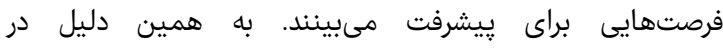

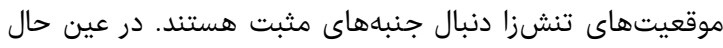

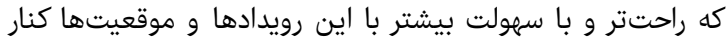

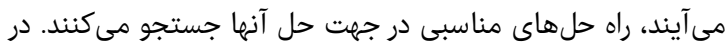

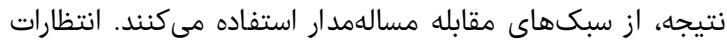

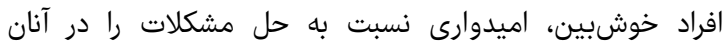

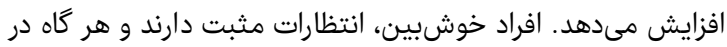

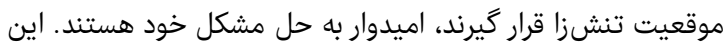

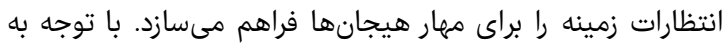

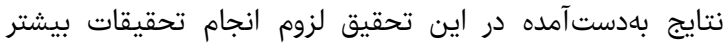
ضرورى بهنظر مىرسد [30].

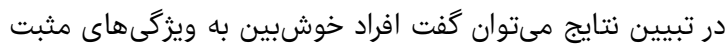

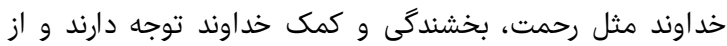

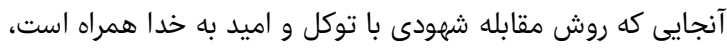

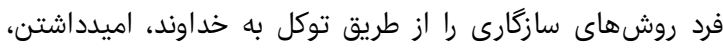

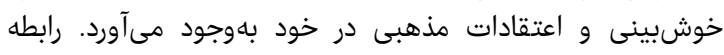

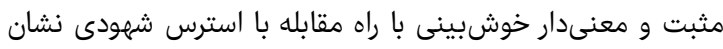

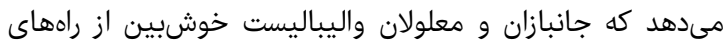


championship level. Ann Appl Sport Sci. 2013;1(1):23-7.

18- Scheier MF, Carver CS. Optimism coping, and health: Assessment and implications of generalized outcome expectancies. Health Psychol. 1985;4(3):219-47.

19- Scheier MF, Carver CS. On the power of positive thinking: The benefits of being optimistic. Curr Dir Psychol Sci. 1993;2(1):26-30.

20- Taati F, Shekari O, shahidi SH. Optimism mediating role in the relationship between educational experience and respond to these stressful experiences. Contemp Psychol. 2013;8(1):73-90. [Persian]

21- Mousavi Nasab SM, Taghavi SM. Optimismpessimism and coping strategies: prediction of psychological adjustment in adolescents. Iran J Psychiatry Clin Psychol. 2007;12(4):380-9. [Persian]

22- Davari R. The relationship between creativity and emotional intelligence to cope with stress styles. Andishe va Raftar. 2007;2(6):49-62. [Persian]

23- Khodayari Fard M, Ghobari Bonab B, Shokouhi Yekta $M$. The domain of psychological researches on religion. Iran J Psychiatry Clin Psychol. 2001;6(4):45-53. [Persian] 24- Madrigal L, Hamill S, Gill DL. Mind over matter: The development of the mental toughness scale (MTS). Sport Psychol. 2013;27(1):62-77.

25- Newland A, Newton M, Finch L, Harbke CR, Podlog L. Moderating variables in the relationship between mental toughness and performance in basketball. J Sport Health Sci. 2013;2(3):184-92.

26- Levy A, Nicholls A, Polman R. Cognitive appraisals in sport: The direct and moderating role of mental toughness. Int J Appl Psychol. 2012;2(4):71-6.

27- Campbell E, Jones G. Sources of stress experienced by elite male wheelchair basketball players. Adapt Phys Activ Q. 2002;19(1):82-99.

28- Zomorrodi S. The relationship between optimism and the secretion of cortisol in male and female students at Allameh Tabatabai University in Tehran [Dissertation]. Tehran: Allameh Tabatabai University; 2007. [Persian]

29- Goudarzi MA, Moeini Roudbali Z. The relationship between coping style and mental health among high school students. Daneshvar Raftar. 2006;3(9):23-32. [Persian]

30- Radi H, Mashayekhi S, Noori N. An inquiry into the relationship of islamic optimism with the depression and ways of coping with the stress among university students. Ravanshenasi va Din. 2012;6(1):31-64. [Persian] investigation of elite sport performers. I Appl Sport Psychol. 2002;14(3):205-18.

4- Kaiseler M, Polman R, Nicholls A. Mental toughness, stress, stress appraisal, coping, and coping effectiveness in sport. Personal Individ Differ. 2009;47(7):728-33.

5- Clough P, Earle K, Sewell D. Mental toughness: The concept and its measurement. In: Cockerill I, editor. Solutions in sport psychology. London: Thomson Publishing; 2002. pp. 32-45

6- Nicholls AR, Polman RC, Levy AR, Backhouse SH Mental toughness, optimism, and coping among athletes. Person Individ Differ. 2008;44(5):1182-92.

7- Besharat M. Psychometric properties of the sport stress coping styles scale. Olympics. 2007;15(3):95-105. [Persian]

8- Hystad SW. Exploring gender equivalence and bias in a measure of psychological hardiness. Int J Psychol Stud. 2012;4(4):69-79.

9- Nasir M. The relationship between coping strategies and resilience and optimism in the city of Dezful secondary school students. J Soc Psychol. 2010;5(16):5568. [Persian]

10- Gould D, Dieffenbach K, Moffett A. Psychological characteristics and their development in Olympic champions. J Appl Sport Psychol. 2002;14(3):172-204.

11- Nes LS, Segerstrom SC. Dispositional optimism and coping: A meta-analytical review. Pers Soc Psychol Rev. 2006;10(3):235-51.

12- Gilak M, Mohammadi A, Bagheri B. The relationship of resiliency and self-concept with self-efficacy of handicapped females: the mediating role of creativity. Dev Pschol. 2013;9(35):307-15. [Persian]

13- Aslankhan MA, Abdoli B, Zamani Sani SH, Fathi Rezaie Z. Emotional intelligence in athletes disabilities. J Iran Psychol. 2009;6(21):15-24. [Persian]

14- Penna P. G, Burden S. A, Richard G. E. Are elite athletes with disabilities mentally tougher than ablebodied competitors? Proceedings of the $3^{\text {rd }}$ International Biennial SELF Research Conference. Sydney: University of Western Sydney; 2004

15- Kashani V. Comparing men and women athletes mentally tough individual contact and non-contact disciplines at different levels of skill [Dissertation]. Tehran: University of Tehran; 2010. [Persian] 16- Yoo J. Factorial validity of the coping scale for Korean athletes. Int J Sport Psychol. 2000;31(3):391-404.

17- Tasaddoghi $Z$. The styles of coping with stress in team and individual athletes based on gender and 